\title{
Current key priorities for the rare diseases patients community in the $\mathbf{E U}$
}

\author{
Flaminia Macchia-Bangsgaard* and Yann Le Cam \\ EURORDIS, Paris, France
}

Rare diseases patients face a lack of access to correct diagnosis, lack of information and public awareness, lack of scientific knowledge and expertise, lack of research, lack of therapeutic development, lack of appropriate healthcare, high cost for most of few existing drugs, inequalities in access to treatment and care, and lack of specialised social services. Because of their rarity these patients are a particularly vulnerable segment of the EU population which disserves a European wide strategy based on social justice and equity.

Therefore, key priorities for rare diseases patients are issues that are generally taken for granted for patients suffering from more common diseases, such as getting an adequate diagnosis relatively quickly, being offered a range of treatments from which they can chose - together with their knowledgeable doctor - the most appropriate one for their specific case, and hopefully being cured or being able to manage their disease towards an improvement of their condition. For most rare diseases patients, this is not the case. Recently, this situation linked to an overall lack of public awareness has started to change and improve, mainly thanks to the relentless work of patient groups and fruitful partnering amongst all interested parties.

\section{Rare diseases: Differences and commonalities}

According to the EU definition, rare diseases affect less than 1 in 2,000 citizens, but the vast majority of rare disease patients suffer from a disease affecting only hundreds or even dozens of patients in the whole of Europe, exacerbating the consequences of rarity. Nevertheless, it is estimated that approximately 30 million people have a rare disease in the EU. Therefore, it is not that unusual to have a rare disease.

Despite their wide diversity, rare diseases have some major common features leading to similar consequences for people living with a rare disease: they are severe to very severe, chronic, often degenerative and life threatening; they are disabling, the lack of autonomy affecting the quality of life; they are highly painful in terms of

\footnotetext{
${ }^{*}$ Corresponding author. E-mail: fmacchia@ @eurordis.org.
} 
psychological burden, aggravated by a feeling of loneliness and despair as well as by lack of support for everyday life; most of them are incurable and they are all difficult to manage.

\section{Patient advocacy: From isolation to patient empowerment}

Currently, EURORDIS, the European Organisation for Rare Diseases, gathers 360 members from 39 countries, 24 of which are EU member states. It is a patient-driven alliance of patient organisations and individuals active in the field of rare diseases. In the last 12 years, since its creation in 1997, EURORDIS and its members have succeeded building a pan-European community of patient organisations and people living with rare diseases.

EURORDIS was founded with the immediate objective to support the adoption of the EU Regulation on Orphan Medicinal Products and has progressively fostered a more favourable environment aimed at improving the life of rare diseases patients and their families, through specific instruments at national and European levels.

\section{Articulating national and European instruments}

Since the entering into force of the Orphan Drugs Regulation in 2000, EURORDIS has led the promotion of rare diseases as a Public Health priority, first at EU level first, thereafter at national level. The definition of this new concept is gradually changing the perception of the public and decision-makers on rare diseases. This shift is reflected into the growing importance given to rare diseases in the Public Health Programme and in the Research and Development Framework Programme of the European Commission. The priority Rare Diseases of the 7th Framework Programme (2007-2013) focuses on Europe-wide studies of natural history and pathophysiology of rare disease as well as on development of preventive, diagnostic and therapeutic interventions. EURORDIS has also played a frontline advocacy role in the adoption of the two recent EU Regulations on Paediatric Drugs and on Advanced Therapies Medicinal Products, expected to boost the development of highly complex drugs and of specific medicines for children.

As promoter, and at times initiator, of reflection processes at European level, EURORDIS is advocating its long-standing proposal for the common scientific assessment the Clinical Added-Value of Orphan Drugs at EU level, where the scarce existing expertise is gathered. This proposal has been included in the recent conclusions and recommendations of the High Level Pharmaceutical Forum on "Improving access to orphan medicines for all affected EU citizens", which aims at supporting the sustainable development of and access to orphan drugs.

In parallel, EURORDIS has been instrumental for the elaboration and adoption by the European Commission in November 2008 of two major documents in the field of 
rare diseases: the "Council Recommendation on a European action in the field of Rare Diseases" and the "Communication on Rare Diseases: Europe's challenges". These documents set the scene for the coming ten years and create the conditions favourable to concrete implementation of solutions for people living with rare diseases in the EU. Furthermore, the Council Recommendation commits all 27 EU Member States to elaborate and adopt National Plan on Rare Diseases. These plans at Member States level will need to be articulated coherently and coordinated efficaciously with all relevant EU policies and strategies, such as the European Reference Networks of Centres of Expertise, Patient Mobility and Cross Border Healthcare.

All these initiatives are inter-connected and underline the specificity of rare diseases, which make them a unique area of high European added-value. 